\title{
The Journal of

\section{Original Article}

\section{Laparoscopic assisted vaginal hysterectomy (LAVH) - An effective alternative to conventional abdominal hysterectomy}

\author{
Kapoor Nisha ${ }^{1}$, Manuja Seema ${ }^{2}$, Mittal Aruna $^{3}$, Gupta Meenakshi ${ }^{3}$ \\ ${ }^{1}$ Senior consultant, ${ }^{2}$ Consultant, ${ }^{3}$ Senior Resident \\ Department of Obstetrics \& Gynaecology, Fortis Escorts Hospital \& Research Center, \\ Faridabad, Haryana, India
}

\begin{abstract}
Objectives: To compare LAVH with TAH in terms of indications, operable uterine size, operative time, intraoperative blood loss, complications, postoperative pain and hospital stay, convalescence and average total cost; also to evaluate LAVH for its feasibility in patients with previous abdominal surgery and those requiring other surgical procedures. Material and Methods: A retrospective study was conducted on 550 patients ( 350 patients of LAVH, 200 patients of TAH) who underwent surgery at Fortis Escorts Hospital, Faridabad, between January 2005 and May 2007. Statistical analysis was done using Student-t test and Chi- square test. Results: On comparing LAVH with TAH, the mean operative time was 63 vs $55 \mathrm{~min}(\mathrm{p}<0.001)$, blood loss 116 vs $150 \mathrm{ml}$ $(\mathrm{p}<0.001)$, major complication rate $2 \%$ vs $5 \%$ hospital stay 2.7 vs 5.7 days $(p<0.001)$. Patients of LAVH had statistically significant lower pain scores, higher activity scores, shorter convalescence and quicker resumption of normal activity. LAVH was marginally costlier than TAH. Conclusion: LAVH enables the surgeon to convert most of the difficult abdominal hysterectomies into vaginal ones with all the benefits of a vaginal procedure. It is also a feasible and safe procedure in patients with previous abdominal surgery, large uteri and adnexal masses. LAVH enjoys patient's support with lesser postoperative pain, shorter hospital stay, rapid return to normal activity and better body image.
\end{abstract}

Key words : LAVH, hysterectomy, laparoscopic, assisted vaginal hysterectomy

\section{Introduction}

Laparoscopic assisted vaginal hysterectomy (LAVH) is becoming increasingly popular among gynecologists and their patients since its advent in 1989. The patient views this endoscopic surgery positively because of the

Paper received on : 25/10/2008 accepted on : 26/12/2009

Correspondence:

Dr. Kapoor Nisha

1691, Sector 16,

Faridabad

Tel no.09818590028

Email: drnishakapoor253@yahoo.com

Fax no: 0129 2426586, 2416260 smaller incisions, faster recovery time and less postoperative pain as compared to a conventional abdominal hysterectomy. For a gynecologist, a combined laparoscopic vaginal approach allows the surgeon to remove larger uteri vaginally, safely tackle abdominal adhesions as in case of previous abdominal-pelvic surgery, and remove endometriosis and complex adnexal masses at the time of vaginal hysterectomy. In addition, it allows one to combine this surgery with other indicated laparoscopic procedures like appendicectomy, cholecystectomy, herniorrhaphy etc. Also, the gynecologist is able to examine the pelvis thoroughly after completion of surgery to ensure complete hemostasis. Finally, the insurance companies and other third party payers are accepting the laparoscopic procedure well in view of a marginally higher cost which is being brought down 
over the years by reducing the hospital stay and using bipolar surgical technique.

\section{Materials and methods}

Between January 2005 and May 2007, 350 laparoscopic assisted vaginal hysterectomies (LAVH) and 200 total abdominal hysterectomies (TAH) were performed at the Fortis Escorts Hospital, Faridabad, Haryana. The patients enrolled for LAVH had selection criteria of uterine size not exceeding 16 weeks and no major cardio-pulmonary disease or advanced gynecological malignancy. Patients not meeting these criteria, or those opting for conventional procedure underwent total abdominal hysterectomy. All the patients undergoing Laparoscopic surgery gave informed consent including the possibility of a laparotomy being necessary for the safe completion of their surgery. This consent included each patient viewing a video teaching film regarding the technique of LAVH.

Pituitary down regulation was done in patients with significant enlarged myomatous uteri and in anemic patients. This allowed for significant reduction in uterine size, making laparoscopic approach easier and safer. In addition, virtual cessation of bleeding allowed for correction of anemia and autologous blood banking.

Preoperatively, patients underwent bowel preparation by taking liquid diet for $24 \mathrm{hrs}$ prior to surgery. Laxative and enema were given the previous evening. All the patients received prophylactic intravenous antibiotic (amoxiclav 1.2gm) $30 \mathrm{~min}$ before surgery. General endotracheal anesthesia was routinely employed and full emergency laparotomy capability was present if required.

A video laparoscopy technique was used with a single or a three chip camera and a television monitor was placed lateral to the patient's feet. A $10 \mathrm{~mm}$ intra-umbilical trocar was inserted after creating pneumoperitoneum. A $10 \mathrm{~mm}$ trocar with inbuilt reduction sleeve on the operator's side and a $5 \mathrm{~mm}$ trocar on the assistant's side were placed under direct vision, midway between symphysis pubis and umbilicus and lateral to the inferior epigastric vessels. An additional $5 \mathrm{~mm}$ suprapubic midline trocar was placed in patients with big uteri or extensive adhesions. An intrauterine manipulator was placed for uterine mobilization during the procedure. Gas insufflation was performed using an automatic $\mathrm{CO} 2$ insufflator. A combination argon beam coagulator / bipolar / unipolar / cautery system was routinely used. LiNA tripol (LiNA Medical Aps, Denmark) was the instrument used for bipolar coagulation and cutting of the vascular pedicles.

Initial careful evaluation of the abdomen and pelvis was performed along with identification of ureters, till their entry into the base of broad ligaments. However no dissection of ureters was routinely performed to reduce operative time and minimize the risk of ureteral vascular compromise. The infundibulo-pelvic, broad and round ligaments were coagulated and cut by Tripol. If the ovaries were to be conserved, the utero-ovarian ligament was dealt with similarly. Vesico-uterine peritoneum was dissected with curved unipolar scissors and the bladder was displaced inferiorly with blunt and sharp dissection. Next, a moist sponge on a ring forceps was placed high up in the posterior fornix and using unipolar hook/scissors, posterior fornix was entered. Anterior fornix was routinely opened vaginally. Bilateral uterine arteries were routinely dealt with via vaginal route. Remainder of the surgery was completed vaginally and vaginal cuff closed by interrupted vicryl no. 1 sutures. A final inspection and irrigation of peritoneal cavity was performed to ensure complete hemostasis. The $10 \mathrm{~mm}$ port sites had absorbable fascial sutures placed in order to minimize the risk of subsequent hernia formation.

We used harmonic blade in patients with extensive adhesions and found it to be of great use due to its cavitational effect, helping in accurate dissection of tissue planes.

Postoperative care included infusing IV fluids and continuous catheterization for $24 \mathrm{hrs}$. Diclofenac rectal suppository was administered at the end of the procedure for pain relief. Early feeding (8-12 hrs postoperatively) was encouraged and the patients were ambulated 12-24 hrs after surgery. The patients were sent home after 48-72 hours with a printed set of instructions. All the patients had a follow up office visit after one and six weeks of surgery.

Statistical evaluation: Student- $t$ test and Chi - square tests were applied to assess the significance while comparing the results of LAVH and TAH. P- value of $<0.005$ was taken as significant.

\section{Observations \& Results}

The age range and parity of our patients is depicted in 
Table 1

\begin{tabular}{|c|c|c|}
\hline \multicolumn{3}{|c|}{ Patient characteristics } \\
\hline Patient characteristics & LAVH & TAH \\
\hline No. of patients & 350 & 200 \\
\hline Mean age (yrs) & $46.2(37-58)$ & $48.3(35-61)$ \\
\hline Parity & $2.3(0-6)$ & $2.8(0-7)$ \\
\hline Mean uterine size (wks) & $12.8(6-16)$ & $13.6(8-20)$ \\
\hline Previous surgery & $112(32 \%)$ & $70(35 \%)$ \\
\hline \multicolumn{3}{|l|}{ Concomitant surgeries } \\
\hline Oophorectomy & $176(50.3 \%)$ & $94(47 \%)$ \\
\hline Adhesiolysis & $100(28.6 \%)$ & $62(31 \%)$ \\
\hline Pelvic floor repair & $28(8 \%)$ & $6(3 \%)$ \\
\hline Appendicectomy & $10(1.7 \%)$ & $2(1 \%)$ \\
\hline Cholecystectomy & $32(9.1 \%)$ & Nil \\
\hline Herniorrhaphy & $28(8 \%)$ & $4(2 \%)$ \\
\hline
\end{tabular}

Table 1. Mean uterine size was 12.8 weeks in LAVH group as compared to 13.6 weeks in the TAH group. The upper limit of uterine size in LAVH patients was 16 weeks. Among the patients who underwent LAVH, 112 $(32 \%)$ had a history of previous abdomino-pelvic surgery as compared to $70(35 \%)$ patients undergoing TAH. Those with previous history of emergency surgery for conditions like intestinal obstruction, ruptured viscera or peritonitis due to any cause were excluded from the laparoscopic surgery group.

Concomitant surgery was performed in both the groups as depicted in Table 1. Surgeries like pelvic floor repair, cholecystectomy and mesh hernioplasty were more readily accepted by the patients with laparoscopic approach. More patients in the TAH group required ad- hesiolysis than in LAVH group on account of the higher number of patients with previous abdominal surgery in the former group.

The indications for performing hysterectomy were comparable in both the groups, the most common indication being fibroid uterus. Other indications were dysfunctional uterine bleeding, adenomyosis, endometriosis, adnexal masses and chronic pelvic inflammatory disease (Table 2). All specimens were routinely sent for pathological examination. Most common pathological diagnosis was myoma(s).

The mean operative time in LAVH group was 63 min (range 45-91min); In TAH group mean time taken to perform the surgery was $55 \mathrm{~min}$ (range $38-87 \mathrm{~min}$ ) and

Table 2

Primary indications for surgery

\begin{tabular}{l|c|c}
\hline Indications & LAVH & TAH \\
\hline Fibroids & $157(44.9 \%)$ & $87(43.5 \%)$ \\
DUB & $60(17.1 \%)$ & $32(16 \%)$ \\
Adenomyosis & $28(8 \%)$ & $13(6.5 \%)$ \\
Postmenopausal bleeding & $7(2 \%)$ & $4(2 \%)$ \\
Chronic cervicitis / Dysplasi & $7(2 \%)$ & $5(2.5 \%)$ \\
Chronic PID & $6(1.7 \%)$ & $5(2.5 \%)$ \\
Endometriosis & $43(12.3 \%)$ & $33(16.5 \%)$ \\
Adnexal masses & $42(12 \%)$ & $21(10.5 \%)$ \\
\end{tabular}


Table 3

Results

\begin{tabular}{|c|c|c|c|c|}
\hline LAVH & TAH & $\begin{array}{c}\mathrm{P} \\
\text { VALUE }\end{array}$ & $\mathrm{SS}^{*} / \mathrm{NS}^{* *}$ & \\
\hline Pain score & $2.083 \pm 0.8107 * * *$ & $7.645 \pm 1.0144$ & $<0.001$ & SS \\
\hline Activity at $1 w k$ & $7.974 \pm 0.6745$ & $3 \pm 0.9327$ & $<0.001$ & SS \\
\hline Activity at $6 \mathrm{wk}$ & $10 \pm 0.0535$ & $6.04 \pm 1.0623$ & $<0.001$ & SS \\
\hline Hospital stay & $2.74 \pm 0.5993$ & $5.71 \pm 1.0936$ & $<0.001$ & SS \\
\hline Operating Time & $63.014 \pm 12.83$ & $54.87 \pm 13.199$ & $<0.001$ & SS \\
\hline Blood Loss (ml) & $116.086 \pm 14.2368$ & $150.2 \pm 24.8387$ & $<0.001$ & SS \\
\hline Need for BT & 42 & 30 & $<0.3167$ & NS \\
\hline Major complications & 7 & 10 & 0.0505 & NS \\
\hline Minor complications & 42 & 40 & 0.0113 & SS \\
\hline $\begin{array}{l}* \mathrm{SS}-\text { Statistically significant } \\
* * \mathrm{NS}-\text { Not statistically signif } \\
* * * \text { Mean } \pm \text { S.D }\end{array}$ & & & & \\
\hline
\end{tabular}

the difference in operative time was statistically significant $(p<0.001)$. As the operations were done in a teaching hospital allowing the residents to observe and assist the laparoscopic procedures, the operative time was prolonged to some extent in cases of LAVH. Also the time taken for surgery grew shorter as more experience with laparoscopy was gained. The mean blood loss during LAVH as determined by the anesthesiologist was $116 \pm 7 \mathrm{ml}$ vs $150 \pm 8 \mathrm{ml}$ in TAH (Table 3 ) and was significantly less in the LAVH group $(\mathrm{p}<0.001)$. The blood loss was calculated accurately by subtracting the volume of irrigation fluid from the amount of fluid collected in suction apparatus. Though $12 \%$ of the patients of LAVH group and $15 \%$ of the patients of TAH group required blood transfusion, the need for blood transfusion was mostly confined to anemic patients preoperatively. Postoperative blood transfusion was required only in $1 \%$ of patients in both the groups $(\mathrm{p}<0.3167)$.

The median length of hospital stay after abdominal hysterectomy was three days longer than that for laparoscopic assisted vaginal hysterectomy and this was statistically significant $(\mathrm{p}<0.001)$. Abdominal hysterectomy was significantly more painful than LAVH, the pain scores being much higher when measured $48 \mathrm{hrs}$ after the procedure, thereby making the analgesia requirement higher $(\mathrm{p}<0.001)$. On the other hand patients of LAVH had significantly higher activity scores at one and six weeks after surgery as compared to conventional abdominal procedure $(\mathrm{p}<0.001)$. The laparoscopic hysterectomy patients required fewer antibiotics as compared to abdominal hysterectomy patients on account of less incidence of febrile morbidity and wound sepsis (Table 4).

In the LAVH group, the major complications included bladder injury in three cases $(0.8 \%)$ of previous LSCS. This was detected preoperatively and repaired laparoscopically. Thermal ureteric injury occurred in one patient $(0.3 \%)$ and was detected 10 days after surgery when the patient presented with left renal pain and hydronephrosis. This was managed by DJ stenting of left ureter for three months. During the study two cases $(0.6 \%)$ were reopened, one each due to omental vessel bleed and uterine artery bleed. Conversion to open surgery was done in two cases $(0.6 \%)$ due to dense adhesions with bowel. One patient out of these two had bowel injury. The complication rate was higher in the first six months of study, which is compatible with the 'learning curve' of any new laparoscopic procedure. The major complication rate was higher in the TAH group $(5 \%)$ but was not statistically significant as depicted in Table $5(\mathrm{p}=0.0505)$.

Minor complication rate including the of wound sepsis, 
Table 4

\begin{tabular}{l|c|c}
\hline \multicolumn{2}{c}{ Results } \\
\hline & LAVH & TAH \\
\hline Hemoglobin & & \\
Preoperative & $10.91 \mathrm{~g} \%$ & $10.80 \mathrm{~g} \%$ \\
Postoperative & $10.53 \mathrm{~g} \%$ & $10.02 \mathrm{~g} \%$ \\
Analgesia & Pethidine pump & Additional analgesics \\
Antibiotics & Amoxyclav X 5 days & $12 \%$ extra antibiotics \\
Cost & Costlier than TAH & \\
\hline
\end{tabular}

Table 5

Results

\begin{tabular}{l|c|c}
\hline & LAVH & TAH \\
\hline Major complications & & \\
Bladder injury & $3(0.8 \%)$ & $2(1 \%)$ \\
Ureteric injury & $1(0.3 \%)$ & $2(1 \%)$ \\
Reopened & $2(0.6 \%)$ & $2(1 \%)$ \\
Bowel injury & $1(0.3 \%)$ & $1(0.5 \%)$ \\
Vault abscess & NIL & $1(0.5 \%)$ \\
Wound dehiscence & NIL & $1(0.5 \%)$ \\
Deep vein thrombosis & NIL & $1(0.5 \%)$ \\
& & \\
Minor complications & $12(3.4 \%)$ & $18(9 \%)$ \\
Fever & $6(1.7 \%)$ & $10(5 \%)$ \\
Wound sepsis & $10(2.9 \%)$ & $6(3 \%)$ \\
UTI & $6(1.7 \%)$ & $6(3 \%)$ \\
Paralytic ileus & $8(2.3 \%)$ & NIL \\
Diaphragmatic irritation & & \\
\hline
\end{tabular}

fever, UTI and paralytic ileus was significantly higher in the TAH patients $(20 \%)$ as compared to patients of LAVH (12\%) (Table 5- $\mathrm{p}<0.0113)$.

\section{Discussion}

In spite of the dramatic increase in the number of LAVH procedures being carried out at various centers, since its first description by Reich ${ }^{1}$, its value remains controversial. Several authors have reported LAVH as an improved alternative to conventional abdominal hysterectomy ${ }^{2,3}$. Other studies have been published discussing LAVH in an outpatient settings ${ }^{2,4}$. Most authors agree that the primary focus and intention of LAVH should be to convert selected abdominal hysterectomies to a vaginal approach thus allowing for surgery in an ambulatory setting. Optimum surgical practice mandates that the severity of the pathologic disorder be the primary criterion in selecting the route of hysterectomy ${ }^{5}$.

Our experience showed that LAVH could be easily performed in a wide variety of patients requiring hysterectomy. Patients prefer this approach because of the far less postoperative pain, smaller abdominal incisions, shorter hospital stay and more rapid return to work or 
other activities. The indications to perform hysterectomy in our study were similar as reported by other authors $^{6,7}$.

The mean operative time taken for LAVH in our study was 63 minutes which is significantly shorter than that reported by other studies ${ }^{8}$. It is acceptable considering the fact that different authors report their series at different stages of learning curve. Moreover, advanced cautery systems, better illumination sources and introduction of harmonic scalpel have considerably reduced the operative time over the last decade. The operative time is also affected by adhesions, increased uterine size and abesity ${ }^{9}$. The average intraoperative blood loss of $116 \mathrm{ml}$ in the LAVH group was comparable to $100 \mathrm{ml}$ reported by Galen 2 and $135 \mathrm{ml}$ reported by Johns 6 but much lower than $390 \mathrm{ml}$ and $417 \mathrm{ml}$ reported by other studies ${ }^{10,11}$. The variations in blood loss and operative time reported in the different studies can be explained by difference in mean uterine size, stage of learning curve and technique.

Postoperatively, pain scores assessed 48 hrs after the procedure were much higher for TAH as compared to the LAVH patients in our study. Similar results have been reported by other authors.

The activity score assessed at one and six weeks postoperatively was considerably higher in the LAVH group vs the TAH group; comparable activity score has been reported in other studies.

Mean hospital stay of 2.7 days in the LAVH group was much less than 5.7 days in the TAH group. Other authors have reported hospital stay varying from $23 \mathrm{hrs}$ to 2.5 days. The length of the hospital stay not only varies from center to center but is also influenced by factors like concomitant surgery etc.

The incidence of major and minor complications was higher in the TAH group as compared to the LAVH group. The postoperative major complication rate of $2 \%$ in our study is comparable to $1.7 \%$ reported by Marana et $\mathrm{al}^{12}$. However it is significantly lower than $8.6 \%$ reported by one study ${ }^{10}$ and $22 \%$ reported by another study ${ }^{13}$.

Most of the patients accepted concomitant surgery more readily with LAVH as compared to open surgery due to cosmetic reasons. However critics of LAVH point to a prolonged operative time, higher incidence of complications and higher cost as compared to TAH. In our study the operative time did shorten as the surgical team gained more experience, which is compatible with the 'learning curve' of any new procedure. In fact as we gained more experience at our center, the operative time between the two approaches was not much different. Similar observation have been made by Seow et $\mathrm{al}^{14}$ in their study, showing reduced complication and conversion rate and decreased operative time on comparing first four years and subsequent four years of the study. The higher cost of laparoscopic surgery is easily offset by shortened hospital stay, better cosmetic outcome, lesser postoperative pain and earlier return to work. Cost can further be curtailed by using bipolar non-staple technique.

\section{Conclusion}

LAVH is a true advance in gynecological surgery since it reduces perioperative morbidity, postoperative pain, intraoperative blood loss and complication rates. Hence it is more acceptable to both - the patients and the gynecologists. In experienced hands, most of the abdominal hysterectomies can easily be converted to vaginal route even in patients with previous abdominal surgeries, large uteri and complex adnexal masses. Higher costs and learning curve are the major constraints at present, which is future hopefully would be taken care of, as has happened in other laparoscopic procedures.

\section{Reference}

1. Reich H, De Caprio J, McGlynn F. Laparoscopic hysterectomy. J Gynecol Surg 1989;5:213-7.

2. Galen D, Jacobson A, Weckstein LN. Outpatient laparoscopic hysterectomy -a review of 50 patients. Paper presented at AAGL 22nd Annual Meeting, San Francisco, 1993.

3. Nezhat F, Nezhat C, Gordon S et al. Laparoscopic versus abdominal hysterectomy. J Reprod Med 1992;37:247-50.

4. Summitt RL Jr, Stovall TG, Lipscomb GH, et al. Randomized comparison of laparoscopy-assisted vaginal hysterectomy with standard vaginal hysterectomy in an outpatient setting. Obstet Gynecol 1992;80: 895-901.

5. Kovac SR. Guidelines to determine the role of laparoscopically assisted vaginal hysterectomy. Am J Obstet Gynecol 1998; 178:1257-63.

6. Johns DA, Diamond MP. Laparoscopically assisted vaginal hysterectomy. J Reprod Med 1994;39:42-8. 
7. Hur M, Kim JH, Moon JS et al. Laparoscopically assisted vaginal hysterectomy. J Reprod Med 1995;40:829-33.

8. Doucette RC, Scott JR. Comparison of laparoscopically assisted vaginal hysterectomy with abdominal and vaginal hysterectomy. J Reprod Med 1996;41:1-6.

9. Chang WC, Lin WC, Hung YC. Pelvic adhesions and laparoscopic assisted vaginal hysterectomy. Int J Gynaecol Obstet 2006;94:145-6.

10. Pelosi MA, Kader N. Laparoscopically assisted vaginal hysterectomy for uteri weighing 500 gm or more. J Am Assoc Gynecol Laparosc 1994;1:405-9.

11. Devendra K, Tay SK. Laparoscopically assisted vaginal hysterectomy - an alternative to abdominal hysterectomy. Singapore Med J 2002;43:138-42.

12. Marana R, Busacca M, Zupi E et al. Laparoscopically assisted vaginal hysterectomy versus total abdominal hysterectomy: a prospective, randomized, multicenter study. Am J Obstet Gynecol 1999;180:270-5.

13. Bornstein SJ, Shaber RE. Laparoscopically assisted vaginal hysterectomy at a health maintenance organization. Cost effectiveness and comparison with total abdominal hysterectomy. J Repord Med 1995;40:435-8.

14. Seow KM, Tsou CT, Lin YH et al. Outcomes and complications of laparoscopically assisted vaginal hysterectomy. Int J Gynaecol Obstet 2006;95:29-34. 